\title{
Applying Computational Science Techniques to Support Adaptive Learning
}

\author{
Juan M. Santos, Luis Anido, Martín Llamas, \\ Luis M. Álvarez, and Fernando A. Mikic \\ E.T.S.E. Telecomunicacións \\ Campus Universitario S/N \\ 36200, Vigo (Pontevedra), Spain \\ \{jsgago, lanido, martin, lmsabu, mikic\}@det.uvigo.es
}

\begin{abstract}
Adaptive Learning Systems offer customized learning experiences according to the actual student needs and capabilities. Effective student modelling, adequate representation of the knowledge domain and proper characterization of learning tools are key issues to provide high quality Adaptive Learning Systems. Most current systems are based on Artificial Intelligence techniques (e.g. fuzzy logic, neural networks, Bayesian networks, etc.) trying to reproduce human teaching behaviours by using a computational representation of expertise. This paper offers a survey on Adaptive Learning showing how Computational Science techniques are applied to instructional systems and identifying forthcoming trends for the future.
\end{abstract}

\section{Introduction}

Information and Communication Technologies (ICT) have been applied to the field of Education during the last years. Advantages of training supported, either completely or partially, by new technologies (commonly called e-learning) have been broadly discussed in many forums. Being aware of these advantages, many institutions both public and private have adopted e-learning in their training and instruction departments.

Web-based e-learning systems have progressively evolved from basic repositories of simple HTML documents to complex learning environments that include tools enabling different educational paradigms like "learning by doing" or "collaborative learning". However, current commercial e-learning systems do not completely make use of all the potential that can be provided by New Technologies. Particularly, most of them do not offer students with learning experiences that are unique and tailored to their needs, interests, preferences, learning style and working environment in order to maximize the effectiveness of learning. Currently, few experimental platforms deal with personalisation and adaptation to each particular student. These platforms, called Adaptive Learning Systems (ALSs), are the result of the effort from researchers that combine two traditionally distinct areas: Instructional Science and Computational Science [1]. 
This paper presents a survey on Adaptive Learning, showing how Computational Science techniques and methods are applied to instructional systems and identifying forthcoming trends. Section 2 deals with the basic concepts involved in the field and presents a common abstract model for ALSs. In Section 3 some examples of currently available ALSs developed by different researching groups are discussed. Finally, Section 4 presents some conclusions and trends for the future.

\section{A Conceptual Model for Adaptive Learning}

According to [2], one of the main reasons to provide adaptation in Web-based e-learning systems is the great variety of users involved. In traditional educational environments, teachers apply a common pedagogical method regardless of the possible heterogeneity among their students. Most conventional systems assume that all the students in the same classroom have acquired the same level of knowledge so far and are prepared to acquire new knowledge at the same level. This is clearly arguable and definitely false in most Internet-based e-learning environments where students may have different background, availability to follow the courses and even a different language and culture.

This situation led researchers in the Artificial Intelligence area to develop Intelligent Tutoring Systems (ITS), which are software tools designed and programmed to "intelligently" reproduce human teaching behaviours by using a computational representation of expertise in instructional methods. For this, they need to model the knowledge domain in question and the learner skills and capabilities. Other aspects subject to adaptation include user interfaces, special requirements for disabled people, etc.

From a Conceptual point of view, an ALS is composed of the Student Model, the Domain Model, the Adaptation Model, the Adaptation Engine, and, in some modern systems, a Environment Model (c.f. Fig. 1):

- Student Model: The Student Model stores relevant information about a particular student and can include, depending on the specific system, his/her personal data, individual preferences, learning style, cultural facets, possible disabilities and background experience and current capabilities for a particular knowledge domain.

- Domain Model: The Domain Model is the repository for storing and structuring the learning contents and the overall knowledge on a particular domain: involved concepts, relevant properties from a pedagogical point of view to study, requirements, goals, etc.

- Environment Model: The Environment Model includes a description of the capabilities of the hardware devices and software applications used by the student in a particular learning session. It can be used to determine the most appropriate form of a resource to be delivered to each particular student's equipment. This model provides ubiquitous access [3] to the learning environment. 


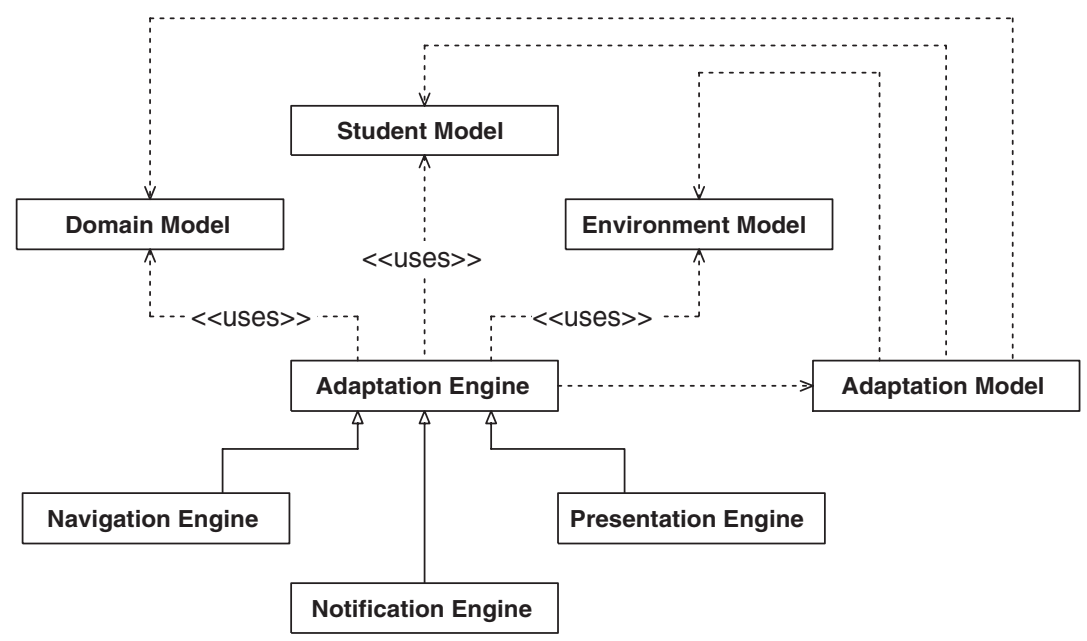

Fig. 1. Conceptual Model for Adaptive Learning Systems

- Adaptation Model: The Adaptation Model contains the specific inferential rules that define how the User Model, Domain Model, and Environment Model elements are combined to provide the actual adaptation.

- Adaptation Engine: The Adaptation Engine is the software process that combines information from the Student, Domain and Environment models and the actual learner interaction with the system in order to personalize the learning process for appropriate learning experiences. It is guided by the rules described in the Adaptation Model. In essence, it is an inference engine, i.e. an automatic control mechanism that applies the axiomatic knowledge present in the knowledge base to arrive at some conclusion: the adequate learning contents to be delivered to the learner.

\section{Computational Science Techniques in ALS}

The development of Adaptive Learning Systems is a highly complex activity. Architects of these sophisticated environments must address with complicated issues like effective student modelling, adequate representation of the knowledge domain, proper characterization of the learning environments or identification and design of appropriate and flexible pedagogical control rules. Many times, it is very difficult, or even impossible, to exactly deal with some of these problems. For example, how can be exactly represented the knowledge level of a student in a particular subject? or, even more, how can be represented such an abstract concept like the student motivation?. Futhermore, the computational complexity of the algorithms needed to automatically take decissions (e.g. which is the most appropiate next lesson for a particular learner) or "on-the-fly" adjust the 
different models from the user interactions with the system are difficult issues to deal with.

Fortunately, it is not necessary to design perfect models and algorithms to get useful adaptive outcomes. For example, it is often better an algorithm that recommends interesting paths in a course organization with a predictive accuracy of $80 \%$ over an algorithm that achieves $90 \%$ if the former requires considerably less CPU time. Modern Adaptive Learning Systems are almost always based on some Artificial Intelligence (AI) technique, like Fuzzy Logic, Neural Networks or, about all, Bayesian Networks.

Bayesian Networks (BNs) are directed acyclic graphs where nodes represent variables and arcs represent probabilistic dependence among variables. These networks, and several variations of them, have steadily been applied in ALSs [4]. They are usually used to represent the student model. Depending on the particular ALS, the variables can be asociated with "concepts", "problems", "abilities", etc, and they are linked by relationships between them, such as "part-of", "prerequisite-of", "evaluation-item-of", etc. A typicall usage setting is showed in Fig. 2.

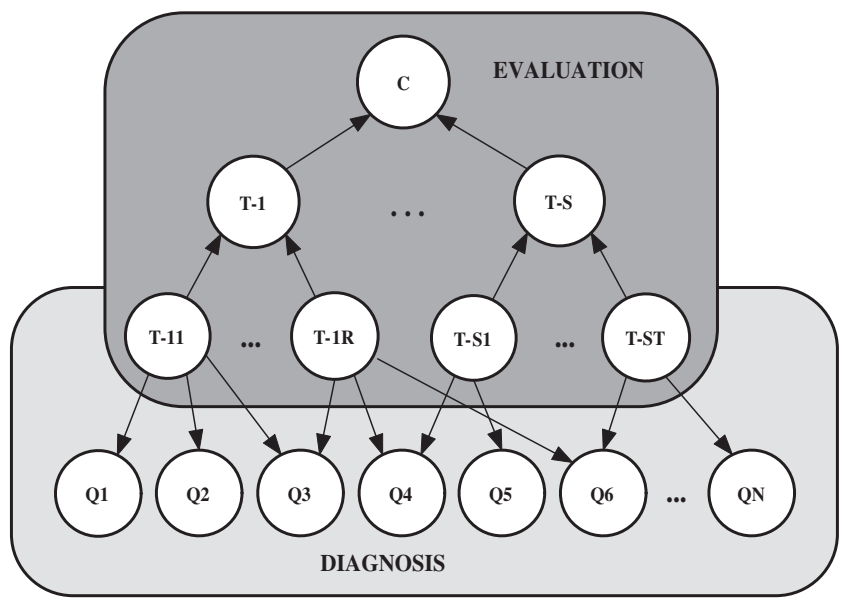

Fig. 2. Use of Bayesian Networks in Adaptive Learnin Systems

In the BN of the figure, upper nodes (Knowledge Nodes) are associated with the topics making up a course, arcs determine the relationship among the different topics and variables of each node indicate the likelihood of the student "knowing" that piece of the course. Bottom nodes (Evidential Nodes) are associated with the test items used to diagnose the knowledge level about the particular topics. Relationships among bottom and upper nodes represent the probabilistic influence of the test items on the Knowledge Nodes. 
To characterize the network it is necessary to define its parameters, i.e., the set of discrete conditional probability distribution of each variable given its parents:

$$
P(X i \mid p a(X i)), i=1, \ldots, n
$$

where $X i$ are the variables and $p a(X i)$ represents the set of the parents of $X i$. This set of probabilities defines the joint probability distribution for the entire network as:

$$
P(X 1, \ldots, X n)=\prod_{i=1}^{n}(X i \mid p a(X i))
$$

These networks are updated in real time, using some approximation algorithm, as the student interact with the learning platform. The information of the student given by the BN is used by the ALS to make different decisions, depending on the particular system. For example, if the BN determines that the student does not understand a topic, a short mini lesson explaining the involved concepts could appear on the user screen.

In the next sub-sections we briefly describe some developed systems, showing the different techniques or approaches used to deal with the different aspects of adaptation.

\subsection{ELM Adaptive Remote Tutor (ELM-ART)}

ELM-ART [5] is an intelligent interactive educational system to support learning programming in LISP. For a student, the system can be viewed as an interactive text-book with support for several types of adaptive navigation, interactive examples and automatic intelligent selection and evaluation of exercises (simple questions and programming problems to solve). It also supports additional functionalities, like a glossary of LISP constructs, a personal annotation tool, a portfolio viewer (where the student can see the set of all analyzed examples and all the solutions of solved problems) or several communication tools.

ELM-ART represents the domain knowledge (Domain Model) in terms of a conceptual network, where units to be learned are organized hierarchically into lessons, sections, subsections and terminal pages (units). Each unit is represented as an object containing slots for the text to be presented to the learner and for information that can be used to relate units to each other (prerequisites, related test items or inference links).

The Student Model is represented as an Overlay Model [6] (where the student' knowledge is considered as a subset of the expert' knowledge) arranged in four layers. The first layer, Visited State, describes whether the user has already visited a unit; the Learner State layer contains information on which exercises related to a particular unit the user has worked at and whether he/she successfully mastered them; the Inferred State layer describes whether a unit could be inferred as known via inference links from more advanced units the user already worked successfully; at last, the Known State, describes whether a unit has been 
marked by the learner as already known. Information in the different layers of the learner model is updated automatically and independently during each interaction with the system. Additionally, students are provided with a tool for inspecting and partially modifying his/her current model.

These models enable both the individual curriculum sequencing and the personalized visual annotation of links. ELM-ART also supports user interface personalization according to the preferences revealed by the students.

\subsection{Intelligent Distributed Environment for Active Learning (IDEAL)}

IDEAL [7] is an intelligent agent-based environment for active learning. The system consists of a number of specialized agents with different expertise. Each student is assigned a unique Personal Agent that manages the student's personal profile (Student Model) including knowledge background, learning styles, interests, courses enrolled in, etc. The Course Agents manage course materials and course-specific teaching techniques for a course. At last, the Teacher Agent interacts with a student and serves as an intelligent tutor of a course. The basic components of a teaching agent are a domain expert module (it creates exercises and questions according to the student's background and learning status, provides solutions and explains the concepts and solutions to cope with student's misconceptions), a pedagogical module (it is a rule-based production system that uses the student model and pedagogical knowledge to determine the appropriate actions) and a student modeller (it provides a model of a student based on his/her learning style, knowledge background and interests).

In IDEAL, the student level of expertise is inferred from the performance of students on exercises and quizzes using a Bayesian belief network. This model incorporates uncertainty to take into account the probability of slips (students sometimes miss questions that they should know) and lucky guesses (correct answers to questions of higher level than student's level). The measure of how well a skill is learned is represented as a probability distribution over skill levels, such as novice, beginning, intermediate, advanced and expert. The conditional probability of skill levels is as follows:

$$
\begin{gathered}
P\left(X=x_{j} \mid Q\right)=\frac{1}{P(Q)} * P\left(X=x_{j}\right) * \\
(1-s)^{\sum_{i=1}^{j}\left(q_{i+}\right)} * s^{\sum_{i=1}^{j}\left(q_{i-}\right)} * g^{\sum_{i=j+1}^{n}\left(q_{i+}\right)} *(1-g)^{\sum_{i=j+1}^{n}\left(q_{i-}\right)}
\end{gathered}
$$

where $X$ represents the skill levels; $Q$ is the evidence vetor of $n$ elements, in which each element $q_{i}$ contains two numbers, $e_{i+}$ and $e_{i-}$, corresponding to the number of correct and incorrect answers to questions at difficulty level $i$ respectively; $s$ is the probability of a slip; and $g$ is the probability of a guess.

The system incorporate a proprietary approach to course content organization and delivery, which is developed based on smart instructional components, 
called lecturelets. Lecturelets contain both the learning resources (concept descriptions, examples, quizzes, etc.) and the instructions on how the resources should be processed or displayed.

\subsection{Self-Paced and Adaptive Courseware (SAC) System}

SAC [8] is a self-paced and adaptive courseware system developed at the Hong Kong Polytechnic University. This system provides dynamic navigational guidance to students taking online courses. It has been designed and implemented on a three-tier web application architecture which uses the Adaptive Hypermedia Application Model (AHAM) [9], a widely used reference model in Adaptive Hypermedia [10] systems.

The AHAM model is composed by tree sub-models:

- The Domain Model describes how the information is structured and linked together in term of concepts and concept relationships.

- The User Model is (conceptually) a table which associate each concept in the Domain Model with a set of attributes. Most of the implementations of this model include the attributes:

- Knowledge Value: it indicates how much the user knows about the concept.

- Read: It indicates whether the user read something (a fragment, a page or a set of pages) about the concept.

A less common attribute would be Ready To Read, which indicates whether the user is ready to access this concept.

- The Teaching Model is a set of pedagogical rules which define how the domain model and the user model are combined to provide ways to perform the actual adaptation. These rules are used also to determine how to compute the attributes of the Domain Model. As an simplified example, the following rule:

$<\operatorname{access}(\mathrm{C} 2)$ AND C1.read=true $\Rightarrow$ C3.ready-to-read=true, true $>$

expresses that when Concept2 is accessed and Concept1 is already read, the Concept3 can be accessed. The true parameter points out that more rules can be processed.

\section{Conclusions and Future Trends}

In spite of the great advantages reported by the researching community, as was mentioned in Section 11, at present, Adaptive Learning Systems have failed to gain widespread acceptance outside the researching laboratories. Thus, the many e-learning platforms that predominate in the current market (such as WebCT [11], IBM LearningSpace [12] or Blackboard Learning System [13]), used by thousands of educational providers, do not offer the possibilities of adaptation 
or personalization depending on the learner's characteristics, or they are very limited. Among the several reasons adduced by the scientists (c.f. [14]), one is widely cited and agreed:

Courses for ALSs are costly to build, needing among 100 and 1000 hours to produce an hour of instructional material.

Solving this problem will be an enormous breakthrough in ALS research. However we want to stand out another critical factor that will determine the final acceptance of these systems: Recently, several organizations and institutions have been thoroughly working towards the development of standards and recommendations aimed to solve the interoperability problems currently found in the e-learning domain [15]. It is foreseeable that near future educational platforms will be developed taken into account the results from this standardization process. Thus, it is essential that both ALS researchers to bear in mind this standardization process and standardization bodies to consider the issues implicated in ALS in order to guaranty the utilization of adaptation techniques.

First proposals from the e-learning standardization process are currently in scene and some manufacturers have begun to adopt them. However, they are not used at all in Adaptive Learning Systems. The reason of this fact resides, on the one hand, in the dedication of ALS researchers to solve particular teaching and pedagogical problems, whithout considering questions such as compatibility. On the other hand, current proposals present some troubles for using them in adaptive environments, being the most outstanding the lack of integration among them (see [16] for a more detailled discusion about this).

Acknowledgments. We want to thank "Ministerio de Ciencia y Tecnología" for their partial support to this work under grant "CORBALearn: Interfaz de Dominio guiada por Estándares para Aprendizaje Electrónico" (TIC2001-3767).

\section{References}

1. Jones, M., Greer, J., Mandinach, E., du Boulay, B., Goodyear, P.: Synthesizing Instructional and Computational Science. In: M. Jones \& P. Winne (eds.): Adaptive Learning Environments: Foundations and Frontiers, pp. 383-401, Berlin: SpringerVerlag (1992)

2. Weber, G.: Adaptive Learning Systems in the World Wide Web. In: J. Kay (Ed.): User modeling - Proceedings of the Seventh International Conference (UM99), pp. 371-378. Vienna: Springer (1999)

3. Dillsus, D., Brunk, C.A., Evans, C. Gladish, B., Pazzani, M.: Adaptive Interfaces for Ubiquitous Web Access. In: Communications of the ACM, Vol 45, No 5. pp. 34-38 (2002)

4. Millán, E., Pérez-de-la-Cruz, J.L.: A Bayesian Diagnostic Algorithm for Student Modeling and its Evaluation. In: User Modeling and User-Adapted Interaction, vol. 12, pp. 281-330, Kluwer Academic Publishers (2002)

5. Weber, G., Brusilovsky, P.: ELM-ART: An adaptive versatile system for Web-based instruction. In: International Journal of Artificial Intelligence in Education, vol. 12, pp. 351-384 (2001) 
6. Van Lehn, K.: Student modelling. In: Foundations of Intelligent Tutoring Systems. Hillsdale, NJ: Lawrence Erlbaum Associates Publishers, pp. 55-76 (1988)

7. Shang, Y., Shi, H., Chen, S.: An Intelligent Distributed Environment for Active Learning. In: ACM Journal of Educational Resources in Computing, vol. 1, issue 2, pp. 308-315 (2001)

8. Chan, A., Chan, S., Cao, J.: SAC: A Self-paced and Adaptive Courseware System. In: Proceedings of the IEEE International Conference on Advanced Learning Technologies (ICALT2001), Wisconsin, U.S.A., IEEE Press (2001)

9. De Bra, P., Houbent, G., Hongjing, W.: AHAM: A Dexter-based Reference Model for Adaptive Hypermedia. In: Proceedings of the 10th ACM Conference on Hypertext and Hypermedia, Darmstadt, Germany, ACM Press (1999)

10. Brusilovsky, P.: Adaptive Hypermedia. In: User Modeling and User-Adapted Interaction, vol. 11, issue 1-2, pp. 87-110, Kluwer Academic Publishers (2001)

11. WebCT web site at http://www.webct.com [last accesed January 1th 2003]

12. IBM' LearningSpace web site at http://www.lotus.com/products/learnspace.nsf/ wdocs/homepage [last accesed on January 1th 2003]

13. Yaskin, D.: Blackboard Learning System (Release 6). Product Overview White Paper. Blackboard Technical Report. Online available at http://products.blackboard.com/cp/release6/LSR6WP.pdf

14. Woolf, B.P., Regian, J.W.: Knowledge-based training systems and the engineering of instruction. In: Training and retraining: A handbook for business, industry, government and the military, pp. 339-356, Macmillan Reference (2000)

15. Santos, J., Caeiro, M., Rodríguez, J., Anido, L.: Standardization in TelE-learning. A Critical Analysis. In: TelE-Learning. The Challenge for the Third Millenium. 17th IFIP World Computer Congress, pp. 321-328, Montreal (Canada). Kluwer Academic Publishers (2002)

16. Santos, J.M., Anido, L., Llamas, M., Rodríguez, J.S.: On the Application of the Semantic Web Concepts to Adaptive E-Learning. In: Shafazand, M. H.; Tjoa, A M. (Eds.) EurAsia-ICT 2002: Information and Communication Technology, Lecture Notes on Computer Science, Vol. 2510, pp. 536-543 (2002) 